\title{
Development of a Polymeric Piezoelectric C-Block Actuator Using Hybrid Optimization Technique
}

\author{
Charles E. Seeley* and Aditi Chattopadhyay ${ }^{\dagger}$ \\ Arizona State University, Tempe, Arizona 85287 \\ and \\ Diann Brei ${ }^{\ddagger}$ \\ University of Michigan, Ann Arbor, Michigan 48109
}

\begin{abstract}
A new class of polymeric piezoelectric bimorph actuators, called C-blocks because of their curved shape, has been developed to overcome limitations of conventional bimorph and stack piezoelectric configurations. Design tradeoffs are investigated in the current research using various performance criteria such as maximum deflection, force, and strain energy. The set of design variables to optimize the C-block actuators, which can be used alone or can be combined in series and/or parallel, includes both continuous and discrete parameters. Therefore, a hybrid optimization technique is developed to address the nonlinear mixed continuous/discrete optimization problem. The results of the optimization procedure indicate useful trends toward microscale actuator development for the most efficient implementation of these actuators.
\end{abstract}

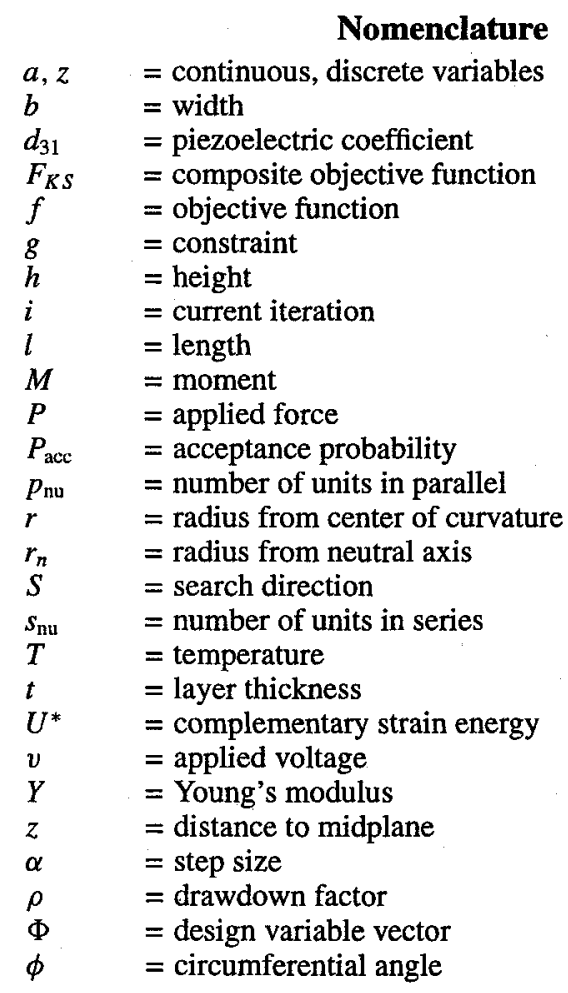

Subscripts

b = bonding layer

$e \quad=$ electrode layer

$L, U \quad=$ lower and upper bounds

Received March 30, 1995; presented as Paper 95-1109 at the AIAA ASME/ASCE/AHS/ASC Structures, Structural Dynamics, and Materials Conference, New Orleans, LA, April 10-12, 1995; revision received July 17, 1995; accepted for publication July 24, 1995. Copyright (C) 1995 by the authors. Published by the American Institute of Aeronautics and Astronautics, Inc., with permission.

${ }^{*}$ Graduate Research Assistant, Department of Mechanical Engineering and Aerospace Engineering. Student Member AIAA.

${ }^{\dagger}$ Associate Professor, Department of Mechanical Engineering and Aerospace Engineering. Senior Member AIAA.

₹Assistant Professor, Department of Mechanical Engineering and Applied Mechanics.

$$
\begin{array}{ll}
\max & =\text { maximum value } \\
n & =\text { neutral axis } \\
p & =\text { piezoelectric layer } \\
\text { tot } & =\text { total structure } \\
1,2,3 & =\text { material coordinates }
\end{array}
$$

\section{Introduction}

Q MART materials have recently found widespread applications in vibration control. The applications range from large flexible space structures, ${ }^{1}$ fixed wing ${ }^{2}$ and rotary wing aircraft, ${ }^{3,4}$ to automotive suspensions. ${ }^{5}$ Piezoelectric materials are popular as actuators because they are light and fast and have low power consumption. They also have a large bandwidth and a relatively large induced strain for an applied voltage. ${ }^{6}$ However, the bimorph and stack configurations most commonly used have limitations insofar as the stack configuration does not produce large deflections and the bimorph configuration supplies only a low force.

A new class of piezoelectric actuators has been developed that demonstrates better force and deflection capabilities. ${ }^{7.8}$ These actuators, called C-blocks because of their half-circle shape, can produce approximately five times the force of the traditional bimorph design with only a slight decrease in deflection. ${ }^{7}$ In addition, C-blocks can be combined in series or in parallel like building blocks to form larger actuator architectures that provide improved deflection or force capabilities, respectively. ${ }^{7,8}$ Because they can be combined, extra design freedoms are gained that create many feasible design options for actuator configurations. With so many alternatives to choose from, selection of the optimal material, geometric and combinatorial parameters for a given application can be difficult. Optimization techniques must be developed to aid in this selection process.

Conventional optimization techniques can be divided into two distinct categories. The first, gradient-based search methods, ${ }^{9}$ rely on gradient information of the objective function and constraints to direct continuous design variables, such as thicknesses or material properties, to improve the objective function. The second category uses combinatorial techniques with discrete design variables, such as number of plies or actuator locations, to improve the objective function. Probabilistic methods ${ }^{10,11}$ are often employed in discrete problems to search for a more global optimum, although often at the expense of additional computational cost. An optimization problem containing exclusively continuous or discrete design variables can be solved using the appropriate technique. Gradient-based methods normally cannot be used to solve a discrete problem unless a continuous relaxation of the discrete variables is possible, which 
may lead to a suboptimal solution due to rounding errors and the possible presence of local minima. Similarly, discrete techniques may be inappropriate for use with continuous problems. The design and optimization of C-block type actuators are associated with both continuous and discrete design variables. Therefore, a hybrid optimization technique ${ }^{12}$ is used that allows inclusion of both types of design variables.

In the current research, a model of the C-block actuators that was developed is extended by combining identical actuators in series or in parallel to improve performance. A hybrid optimization technique developed by Chattopadhyay and Seeley ${ }^{12}$ is used that is based on a modified simulated annealing (SA) algorithm ${ }^{13,14}$ and utilizes available gradient information to improve efficiency. The procedure is applied to obtain design trends for optimal C-block actuator configurations that are still in the conceptual design phase.

\section{Mathematical Model}

In the following sections, analytical models for single C-block actuators are developed that are extended to include advanced actuator architectures. Experimental validation is then provided to ensure the accuracy of the analysis. Tradeoffs between the C-block actuators and conventional straight bimorph actuators are also discussed.

\section{A. Analytical Analysis}

The C-block actuators are constructed out of two curved piezoelectric layers in a bimorph configuration resulting in a halfcircle as shown in Fig. 1. The piezoelectric material is chosen to be polyvinylidene fluoride (PVDF) since piezoceramic materials are too brittle to withstand the necessary deformations required and are too awkward to form into unusual shapes. The piezoelectric layers are surrounded on both sides by an electrode layer of silver ink. The bonding layer consists of a common epoxy used to connect the two piezoelectric layers. These layers are actuated with equal but opposite electric fields to produce a bending moment that is used as the actuation mechanism. The equations for the $\mathrm{C}$-block actuator, which is modeled as a curved beam, have been developed for the most part in a previous work. 7,8 A Bernoulli-Euler strain distribution is assumed instead of a uniform strain distribution since the ratio of total actuator thickness to the piezoelectric material thickness is small (less than five). ${ }^{15}$ An energy method is used to derive the deflection and force models of the C-block actuator.

The moment created by energizing the piezoelectric layer $\left(M_{e}\right)$ for a single $\mathrm{C}$-block actuator is determined as follows ${ }^{7}$ :

$$
M_{e}=Y_{p} d_{31} E_{3} b_{p}\left(z_{e}^{2}-z_{p}^{2}\right)
$$

where $E_{3}$ is the applied electric field, and $z_{e}$ and $z_{p}$ are the distances from the electrode and piezoelectric layers to the midplane, respectively. The complementary strain energy $U^{*}$ is calculated by first determining the total bending moment $M$, which includes the moment due to the piezoelectric actuation $M_{e}$ and the moment due to any external loads. Then integration over the cross-sectional area, including all of the piezoelectric, electrode, and bonding layers, yields in the following expression

$$
U^{*}=\int \frac{M^{2}}{2\left(r-r_{n}\right) Y A_{\mathrm{tot}}} \mathrm{d} \phi
$$

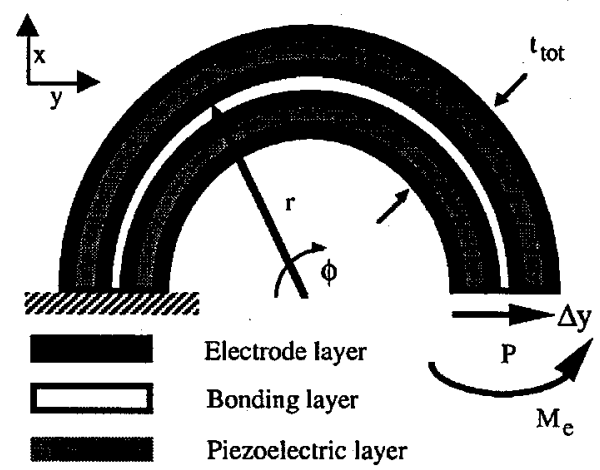

Fig. 1 Single C-block actuator.

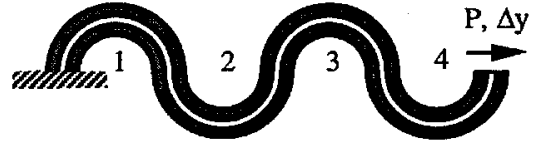

Fig. 2 C-block actuators combined in series.

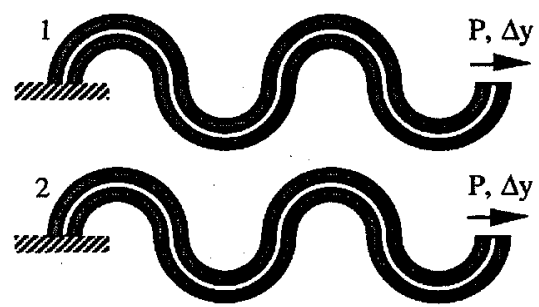

Fig. 3 C-block actuators combined in series and in parallel.

where the quantities $r$ and $r_{n}$ are the distances to the midplane and neutral axis, respectively, $Y A_{\text {tot }}$ represents the stiffness of the actuator, and $d \phi$ is the differential circumferential angle. The formulations of $r_{n}$ and $Y A_{\text {tot }}$ are somewhat lengthy and can be found in Ref. 7. It is assumed that no external loads are applied so that $M=M_{e}$. The deflection for a C-block actuator is determined by using Castigliano's theorem. By adjoining several C-block actuators in series, as shown in Fig. 2, and assuming that all of the C-block units are identical and are energized in the same manner, the total deflection is written as follows:

$$
\Delta_{y}=s_{\mathrm{nu}} \frac{2 M_{e} r}{Y A_{\mathrm{tot}}\left(r-r_{n}\right)}
$$

Similarly, by using Castigliano's theorem and combining several C-block actuators in parallel as indicated in Fig. 3, the induced force $P$ from piezoelectric actuation at the tip of the actuator is as follows:

$$
P=-p_{\mathrm{nu}}\left(4 M_{e} / r \pi\right)
$$

Applications of these C-block actuators may require a combination of both deflection and force. The most efficient representation of both the deflection and the force together is the work done by the actuator or, equivalently, the strain energy induced by piezoelectric actuation. The strain energy $U^{*}$ produced by a number of C-block actuators combined in both series and in parallel is found by integrating of Eq. (2) over all units in series and parallel as follows:

$$
U^{*}=p_{\mathrm{nu}} s_{\mathrm{nu}} \frac{M_{e}^{2} \pi}{2\left(r-r_{n}\right) Y A_{\mathrm{tot}}}
$$

As shown in the preceding analysis, the deffection, force, and work done by the C-block actuators can be significantly increased by combining multiple units in series, parallel, or both. In the following sections, experimental validation is provided for the mathematical models presented and the hybrid optimization technique that is used to determine design trends for the C-block actuators is described.

\section{B. Experimental Validation}

A variety of experiments are performed to validate the mathematical models for the polymeric C-block actuators. A high voltage supply is connected directly to a clamped C-block prototype for actuation. A three-dimensional scale is used for deflection measurements, and force measurements are performed by hanging small aluminum weights from a loop of thread attached to the tip of the C-block. Further details of the experimental setup can be found in Ref. 7. Three different types of experiments are performed: deflection voltage, force voltage, and force deflection. Each experiment is replicated with a wide variety of prototypes that differed in radii, layer thicknesses, and the number of C-blocks used in combination. Representative results for each experiment are presented next.

\section{Deflection Voltage Experiment}

The deflection voltage experiments are used to test the maximum deflection generated by $\mathrm{C}$-blocks for a given input voltage. In this 


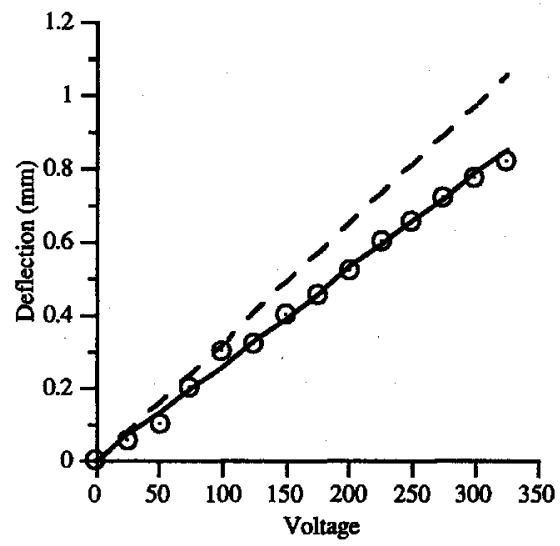

Fig. 4 Single actuator; deflection vs voltage $\left(r=1.37 \mathrm{~cm}\right.$ and $t_{\text {tot }}=$ $0.100 \mathrm{~mm}):-$, analytical C-block; $\odot$, experimental C-block;... , analytical straight bimorph.

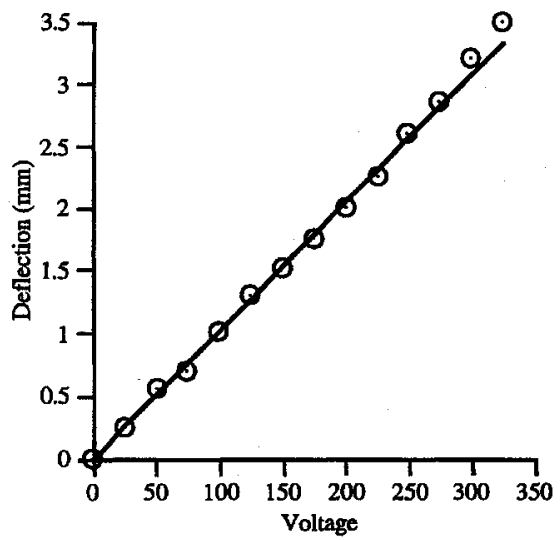

Fig. 5 Series actuator; deflection vs voltage $\left(r=1.39 \mathrm{~cm}\right.$ and $t_{\text {tot }}=$ $0.112 \mathrm{~mm})$ : - , analytical C-block; $\odot$, experimental C-block.

experiment, a dc voltage is applied to the actuator in steps of $25 \mathrm{~V}$ from zero to $325 \mathrm{~V}$. For each voltage increment, the coordinate of the tip is measured and the initial position is subtracted to determine the deffection. The analytical and experimental results for a representative single $\mathrm{C}$-block actuator are shown in Fig. 4 along with the results for an equivalent PVDF straight bimorph presented in Ref. 16. The deflection of the C-block actuator is slightly less compared with the conventional straight bimorph. A serial actuator with two units in series are shown in Fig. 5 where the performance of the C-block actuator is significantly improved by combining units in series. The results from all of the experiments are within $10 \%$ of the predicted behavior for each set of radii and thickness parameters. These results validate the mathematical models and prove that the deflection from individual C-blocks add linearly.

\section{Force Voltage Experiment}

The force voltage experiments are used to test the maximum force generated by $\mathrm{C}$-blocks for a given input voltage. Again, a dc voltage is applied in $25-\mathrm{V}$ increments up to a maximum of $325 \mathrm{~V}$. For each voltage increment, the force is measured by adding weights to the thread loop until the tip deflection is zero. The results for a representative C-block are shown in Fig. 6. The experimental and theoretical results are in excellent agreement, which validates the quantitative models. A representative PVDF straight bimorph is also presented in Fig. 6. It can be seen that the force generated by a basic C-block element is significantly larger than that generated by a conventional straight bimorph.

\section{Force Deflection Experiment}

The force deflection experiments are used to test the overall static performance of C-block actuators. A constant voltage of $325 \mathrm{~V}$ is applied to a single C-block actuator, and the tip deflection is determined. Weights in increments of $20 \mathrm{mg}$ are then added to the thread loop. The tip deflection at each additional weight is also determined.

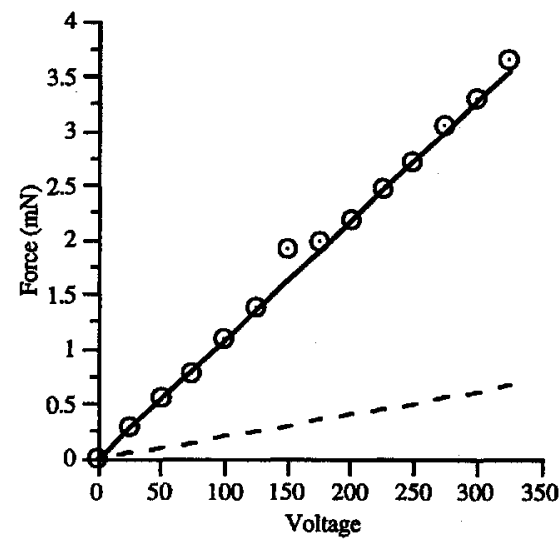

Fig. 6 Single actuator; force vs voltage $\left(r=1.56 \mathrm{~cm}\right.$ and $t_{\text {tot }}=$ $0.148 \mathrm{~mm}$ ): - , analytical C-block; $\odot$, experimental C-block; ..., analytical straight bimorph.

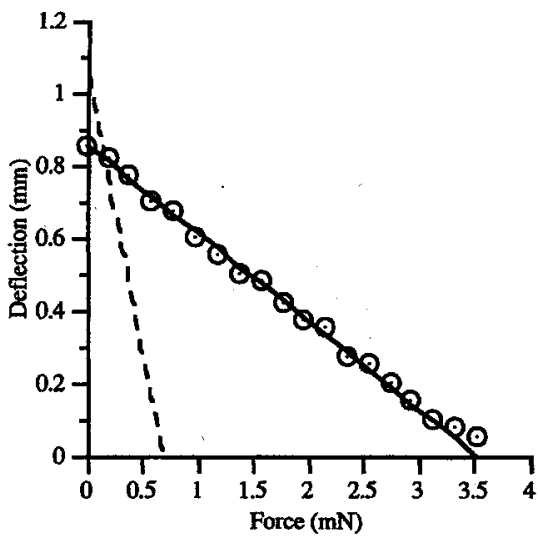

Fig. 7 Single actuator; force vs deflection $\left(r=1.56 \mathrm{~cm}\right.$ and $t_{\text {tot }}=$ $0.148 \mathrm{~mm}):-$, analytical C-block; $\odot$, experimental C-block; ...., analytical straight bimorph.

This procedure is repeated until the deflection of the tip is zero. The results for a representative C-block are shown in Fig. 7. Once again, excellent agreement is observed between theory and experiment. The representative PVDF straight bimorph, also presented in Fig. 7, indicates that $\mathrm{C}$-block actuators generate significantly larger force but less deflection than a straight bimorph.

\section{Optimization Formulation}

A general single objective optimization problem involves the minimization of a single objective function, $f(\Phi)$, subject to a set of constraints, $g_{n}(\Phi)(n=1,2, \ldots, \mathrm{NCON})$, where NCON is the number of constraints. Side constraints, $\Phi_{L} \leq \Phi \leq \Phi_{U}$, are also imposed on the design variables. The design variable vector $\Phi$ includes both continuous design variables $a_{i}$ such as the piezoelectric layer thickness $t_{p}$ and discrete design variables $z_{j}$ such as the number of actuators in parallel $p_{\text {nu }}$ (Figs. 1-3). The use of continuous design variables with combinatorial techniques can be computationally very expensive, if possible at all. Similarly, discrete variables are not compatible with gradient-based optimization methods unless a continuous relaxation of the discrete variables is allowed, which may lead to suboptimal solutions. Few formal methods are available for solving nonlinear optimization problems that contain both discrete and continuous design variables. Therefore, a new hybrid technique developed by Chattopadhyay and Seeley ${ }^{12}$ is used that effectively improves the objective function while satisfying all constraints and incorporating both types of design variables.

The hybrid optimization procedure ${ }^{12}$ requires that a single objective function be minimized. Therefore, the KreisselmieierSteinhauser (K-S) function approach is used to efficiently combine multiple and conflicting design objectives and constraints into a single composite function. ${ }^{17}$ Recent studies have successfully demonstrated the usefulness of the $\mathrm{K}-\mathrm{S}$ function technique in practical design problems. ${ }^{18}$ Using the $\mathrm{K}-\mathrm{S}$ formulation, the original 
objective function is transformed into a reduced objective function, $f^{*}(\Phi)$, as described in Ref. 18. Since the reduced objective function is analogous to a constraint, a new constraint vector $f_{m}(\Phi)$ is introduced $(m=1,2, \ldots, M$, where $M=\mathrm{NCON}+1)$, which includes the reduced objective function in addition to the original constraints. The new composite $\mathrm{K}-\mathrm{S}$ objective function to be minimized is defined as follows:

$$
F_{K S}(\Phi)=f_{\max }+\frac{1}{\rho} \ln \sum_{m=1}^{M} e^{\rho\left(f_{m}-f_{\max }\right)}
$$

where the quantity $f_{\max }$ represents the maximum reduced objective function or constraint value and the parameter $\rho$ is analogous to the drawdown factor of penalty function formulations and controls the distance from the surface of the $\mathrm{K}-\mathrm{S}$ envelope to the surface of the maximum new constraint function. ${ }^{18}$ A larger value of $\rho$ moves the $\mathrm{K}-\mathrm{S}$ function envelope closer to the maximum constraint, whereas a smaller value of $\rho$ retains contributions from the objective function and constraints. The result is the best feasible compromise between the original objective function and the constraints.

Once the $\mathrm{K}-\mathrm{S}$ function is formulated, the hybrid optimization algorithm is used to minimize $F(\Phi)_{K S}$. The hybrid algorithm uses the SA technique in conjunction with a gradient-based search. This results in a optimization technique that can efficiently include both continuous and discrete design variables. The algorithm is outlined next.

\section{START}

Current design is $F_{K S}$

Select either continuous or discrete variable to perturb for $F_{K S_{\text {new }}}$ If continuous variable selected

$$
a_{i_{\mathrm{new}}}=a_{i}+\alpha S_{i}
$$

Else if discrete variable selected

$$
z_{j_{\mathrm{new}}}=z_{j q}
$$

End if

Compute $F_{K} S_{\text {new }}$

If $F_{K S_{\text {new }}} \leq F_{K S}$, then

$$
F_{K S}=F_{K S_{\text {new }}}
$$

Else if $P_{\text {acc }} \geq P$, then

$$
F_{K S}=F_{K S_{\text {new }}}
$$

End if

\section{Go to START}

In the preceding algorithm, the quantity $F$ is the current value of the $\mathrm{K}-\mathrm{S}$ function $F_{K S}$. The continuous and discrete design variables are represented by $a$ and $z$, respectively, and the subscript "new" denotes current perturbed design variables. The quantity $\alpha$ is a random step size selected to be within some percentage of the current value of the continuous variables (e.g., $-1 \% \leq \alpha \leq+1 \%$ ) and $S_{i}$ is the search direction that is determined from the gradient of the $\mathrm{K}-\mathrm{S}$ composite objective function ${ }^{16}$ for the current design variable. The continuous design variables are perturbed using the step size and the search direction to improve the efficiency of the search whereas the discrete variables are perturbed by randomly selecting specified values $z_{j q}$ within a given range. Move limits and bounds are imposed on the continuous design variables to ensure a physically meaningful design. The parameter $P$ is a random number such that $0 \leq P \leq 1$ and the acceptance probability $\left(P_{\text {acc }}\right)$ of retaining a worse design is computed as follows:

$$
P_{\text {acc }}=e^{-(\Delta F / T)}
$$

where $\Delta F$ represents the change in objective function and $T$ is the temperature that is computed during each iteration using the following relation:

$$
T=T_{0} r^{i}
$$

where the temperature $T$ is reduced from the initial temperature $T_{0}$ for successive iterations $i$ according to the cooling rate $r{ }^{19} \mathrm{~A}$ higher temperature allows worse designs to be occasionally accepted according to the given probability, which allows the algorithm to climb out of possible local minima. The probability is reduced to zero during optimization so that only better designs are accepted to ensure smooth convergence.

The use of the directed search for the continuous design variables increases the efficiency. However, the probabilistic nature of the SA algorithm allows the optimization procedure to climb out of local minima. Therefore, the hybrid procedure exhibits benefits of both gradient-based and discrete optimization techniques.

\section{Results}

The hybrid optimization procedure is implemented along with the C-block actuator models developed to determine design trends for three different cases using three different design objectives. These design objectives include induced deflection, force, and strain energy of the actuators due to activation of the piezoelectric material. The different cases studied are described next.

Maximize case 1 : deflection $\Delta y$, case 2 : force $P$, and case 3 : strain energy $U^{*}$, subject to

$$
\begin{array}{cl}
g_{1}=\left(z_{b} / r\right)-1 \leq 0, & g_{2}=\left(z_{e} / r\right)-1 \leq 0 \\
g_{3}=\left(z_{p} / r\right)-1 \leq 0, & g_{4}=\left(z_{e 0} / r\right)-1 \leq 0 \\
g_{5}=\left(t_{\text {tot }} / r\right)-1 \leq 0, & g_{6}=\left(r_{n} / r\right)-1 \leq 0 \\
g_{7}=\frac{2}{h_{\max }}\left[r+\frac{p_{\text {num }} t_{\text {tot }}}{2}\right]-1 \leq 0 \\
g_{8}=\frac{2 r s_{\text {num }}}{l_{\max }}-1 \leq 0, & g_{9}=\left(v / v_{\max }\right)-1 \leq 0
\end{array}
$$

where the side constraints are

$$
\begin{array}{cc}
5 \leq t_{b} \leq 1000 \mu \mathrm{m}, & 0.6 \leq t_{e} \leq 20.0 \mu \mathrm{m} \\
8.0 \leq t_{p} \leq 1000.0 \mu \mathrm{m}, & 0.001 \leq r \leq 50.0 \mathrm{~mm}
\end{array}
$$

The optimization problem is formulated with four continuous design variables. The first three continuous design variables are the thicknesses of the bonding, electrode, and piezoelectric layers represented by $t_{b}, t_{e}$, and $t_{p}$, respectively, and the radius $r$. Two additional design variables, $p_{\mathrm{nu}}$ and $s_{\mathrm{nu}}$, are discrete variables and represent the number of units in parallel and in series, respectively. The width of the actuator is chosen to be $1 \mathrm{~cm}$ for convenience. All other parameters describing the C-block actuator model are either previously specified depending on the case investigated or can be determined from the six independent design variables.

The first four constraints $\left(g_{1}-g_{4}\right)$ are imposed to ensure that the distance from any layer to the midplane is less than or equal to the radius. The fifth constraint $g_{5}$ guarantees that the total thickness is also less than the radius for a physically meaningful design. A single C-block actuator is permitted to curve only in one direction to avoid numerical problems caused by taking logarithms of negative numbers in the formulation of $r_{n}$ as mandated by the sixth constraint $g_{6}$. In many practical applications, actuators have strict length and height limitations. The seventh and eighth constraints, $g_{7}$ and $g_{8}$, represent length and height constraints, respectively, which are imposed to ensure that the actuator fits inside a specified volume. It is assumed that the maximum possible electric field is applied to the actuators without damage to or depolarization of the piezoelectric layers. Since the voltage required to maintain a constant electric field increases proportionately with the piezoelectric layer thickness, a constraint $g_{9}$ is placed on the maximum applied voltage to ensure a practical design. Lower bounds on the thickness design variables are due to manufacturing limitations. Upper bounds are imposed to ensure a physically reasonable design. The bounds on the radius design variable are also justified in this manner. Side constraints on the discrete design variables are implied when the specific allowable values for each variable are specified. The material and piezoelectric properties and additional parameters are presented in Table 1. 
Table 1 Material properties and miscellaneous parameters

\begin{tabular}{lllc}
\hline \multicolumn{2}{c}{ Material properties } & \multicolumn{2}{c}{ Miscellaneous parameters } \\
\hline$Y_{b}, \mathrm{~N} / \mathrm{m}^{2}$ & $1.9 \times 10^{9}$ & $v_{\max }, \mathrm{V}$ & 50.0 \\
$Y_{p}, \mathrm{~N} / \mathrm{m}^{2}$ & $5.4 \times 10^{9}$ & $E_{3}, \mathrm{~V} / \mathrm{m}$ & $30.0 \times 10^{5}$ \\
$Y_{e}, \mathrm{~N} / \mathrm{m}^{2}$ & $7.0 \times 10^{8}$ & $l_{\max }, \mathrm{cm}$ & 10.0 \\
$d_{31}, \mathrm{~m} / \mathrm{V}$ & $2.3 \times 10^{-11}$ & $h_{\max }, \mathrm{cm}$ & 3.00 \\
\hline \hline
\end{tabular}

Table 2 Optimization results

\begin{tabular}{lcccc}
\hline Design & & \multicolumn{3}{c}{ Final } \\
\cline { 3 - 5 } variable & Initial & Case 1: $\Delta y$ & Case 2:P & Case 3: $U^{*}$ \\
\hline$t_{b}, \mu \mathrm{m}$ & 10.0 & 5.00 & 5.00 & 5.00 \\
$t_{e}, \mu \mathrm{m}$ & 10.0 & 0.60 & 0.60 & 0.60 \\
$t_{p}, \mu \mathrm{m}$ & 10.0 & 8.00 & 16.2 & 16.7 \\
$r, \mathrm{~mm}$ & 0.10 & 15.0 & 0.04 & 0.19 \\
$t_{p} / t_{\mathrm{tot}}$ & 0.14 & 0.34 & 0.41 & 0.41 \\
$p_{\mathrm{nu}}$ & 100 & $(1)$ & 752 & 722 \\
$s_{\mathrm{nu}}$ & 100 & 1 & $(1)$ & 261 \\
$\Delta y, \mathrm{~mm}$ & 0.004 & 3.08 & 0 & 0.076 \\
$P, \mathrm{~N}$ & 1.52 & 0 & 25.41 & 5.50 \\
$U^{*}, \mathrm{Nm}$ & $3.84 \times 10^{-6}$ & $\sim 0$ & $\sim 0$ & $2.59 \times 10^{-4}$ \\
$v, \mathrm{~V}$ & 30.0 & 24.0 & 48.6 & 50.0 \\
$t_{\mathrm{tot}}, \mu \mathrm{m}$ & 70.00 & 23.4 & 39.8 & 40.8 \\
\hline \hline
\end{tabular}

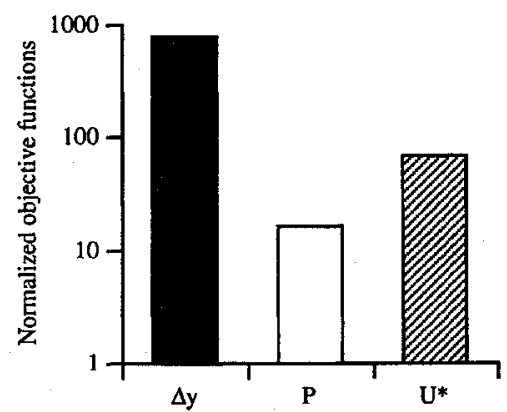

Fig. 8 Normalized objective functions for three cases.

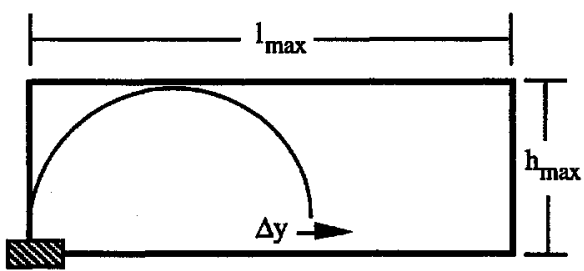

Fig. 9 Single thin actuator to maximize deflection.

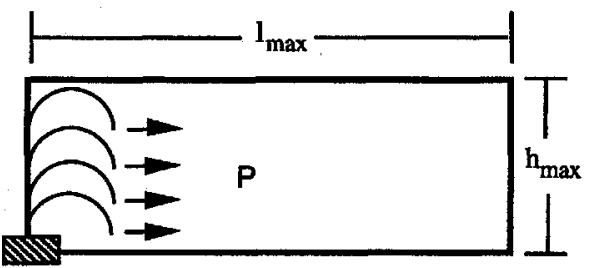

Fig. 10 Multiple actuators in parallel to maximize force.

The results obtained using the hybrid optimization procedure are presented in Table 2 and in Figs. 8-11. Analytical gradients are calculated for the continuous design variables in the current work to reduce computational effort during optimization. A value of $\rho=50$ is used for the $\mathrm{K}-\mathrm{S}$ function to ensure that no constraints are violated in the final design. The hybrid algorithm randomly selects a single design variable, either discrete or continuous, for each iteration. A step size of up to $1 \%$ is found to be appropriate for the continuous variables. The discrete variables can be selected from integer values within a range of $\pm 10 \%$ of their current values. Significant improvements are obtained in all of the objective functions while satisfying the constraints in the final design. The objective functions are

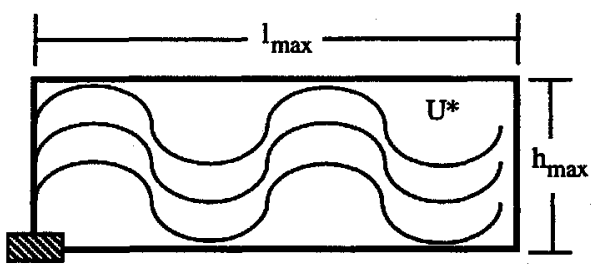

Fig. 11 Multiple C-blocks in parallel and series to maximize strain energy.

presented, normalized with respect to their initial values, in Fig. 8. Note that the objective functions must be scaled to a log axis due to the dramatic improvements after optimization. For instance, the deflection $\Delta y$ increases almost three orders of magnitude compared with the initial design. The respective dimensional values can be found in Table 2. A summary of the optimization results is also presented in Table 2 for the three different cases that are investigated. Design variables that are not relevant for a particular case are held constant during optimization and are indicated in this table by (). It must be noted here that the dramatic improvements are based on a reference configuration that is still in the conceptual phase and needs further development. The purpose of this research is to investigate feasible design trends.

\section{A. Case 1-Maximize Deflection}

In this case, the induced deflection of the actuator is maximized by minimizing $f=-|\Delta y|$. Since the deflection is independent of the number of units in parallel, $p_{\text {nu }}$ is set equal to 1 and is eliminated from the set of design variables. Therefore, constraint $g_{7}$ assumes the following form:

$$
(2 / \bar{h})\left[r+\left(t_{\mathrm{tot}} / 2\right)\right]-1 \leq 0
$$

As both $r$ and $s_{\mathrm{nu}}$ increase, the deflection also increases. Since the length constraint is clearly active in the final design, the optimization problem can be simplified by using the length constraint $g_{8}$ to determine $r$, thereby eliminating it as an independent design variable,

$$
r=l_{\max } / 2 s_{\mathrm{nu}}
$$

The design variables corresponding to the three thicknesses, $t_{b}, t_{e}$, and $t_{p}$, are all driven to their respective lower bounds during optimization. In the final design, $s_{\mathrm{nu}}=1$, indicating that a single thin actuator with radius $r=l_{\max } / 2$ maximizes the deflection. It was previously noted in Refs. 8 and 15 that for a single actuator deflection is maximized by a thin, straight beam with a ratio $t_{p} / t_{\text {tot }}=0.4$. This trend is also observed in the current optimization results where the ratio $t_{p} / t_{\text {tot }}$ approaches 0.4 , although the lower bound imposed on $t_{p}$ is reached before this optimal ratio can be achieved. Additionally, the largest possible radius is obtained, indicating the trend towards a straight beam for this case. Figure 9 presents the representative final design for displacement maximization. The volume constraints are also included in this figure as indicated by the box surrounding the C-block actuator. Note that the deflection is maximized at the expense of the force, thus drastically reducing the available work done by the actuator (Table 2).

\section{B. Case 2-Maximize Force}

This case investigates force as the objective function that is maximized by minimizing $f=-|P|$. In this case, the force is independent of the number of units in series, $s_{\mathrm{nu}}$. Therefore, its value is set equal to 1 and it is eliminated from the design variable vector. The results for this case are presented in Table 2 and Fig. 10. Although the height constraint $g_{7}$ is active in the final design, it is not used to eliminate any design variable as in the previous case due to numerical problems that can develop during optimization such as attempts to take logarithms of negative numbers. The electrode layer thickness $t_{e}$ decreases to its lower bound due to its lower modulus compared with the other layers present. The piezoelectric layer $t_{p}$ increases until the voltage constraint is violated, and the radius $r$ decreases until its value reaches the total thickness of the actuator. The ratio $t_{p} / t_{\text {tot }}$ is also near 0.4 as observed in previous 
work ${ }^{8,15}$ However, contrary to a previous finding ${ }^{15}$ that showed that the bonding layer $t_{b}$ increases to increase the moment arm that maximizes the force in a single actuator configuration, the present study shows that the bonding layer thickness $t_{b}$ reduces to its lower bound, whereas the number of units in parallel $p_{\text {nu }}$ increases until the height constraint becomes active. This indicates that many thin curved beams acting in parallel represent a superior configuration than a single thick curved beam. Although the induced deflection is very small, it must be noted that a large force of $25.4 \mathrm{~N}$ develops from such an actuator configuration (Table 2). The representative actuator that maximizes the force is shown in Fig. 10. Only a single unit in series is presented with many units in parallel since the force is independent of the number of units in series $s_{\mathrm{nu}}$. The optimal design cannot be experimentally tested yet because the large number of very small C-block actuators is beyond the capabilities of current microscale manufacturing technology. However, the design trends for this case suggest that many microscale actuators are more efficient than a few larger actuators. Therefore, further research into microscale technology is mandated.

\section{Case 3-Maximize Strain Energy}

It is desirable to create an actuator that can produce both deflection and force. Therefore, the induced strain energy (work) from piezoelectric actuation is maximized as an objective function by minimizing $f=-\left|U^{*}\right|$. Results for this case are presented in Table 2 and Fig. 11. The results indicate a tradeoff between the those obtained through individual maximization of force (case 1) and deflection (case 2). It is observed from Table 2 that the bonding layer and electrode layer thicknesses decrease to their respective lower bounds, whereas the piezoelectric layer thickness increases until the voltage constraint becomes active. Again, the optimal ratio $t_{p} / t_{\mathrm{tot}}=0.4$ is obtained. The results agree with the optimal values obtained by Crawley and Anderson ${ }^{15}$ for similar structures. Since the bonding layer thickness is driven to its lower bound, the number of units in parallel $p_{\text {nu }}$ increases until the height constraint $g_{7}$ becomes active. Since this constraint is active in the final design, there is a tradeoff between a few thick actuators and many thin actuators to maximize $U^{*}$. The intermediate value of the radius in the final design, compared with the first two cases, is also an indication of this tradeoff. The hybrid optimization technique indicates that many thin actuators in parallel are superior to a few thick ones (Fig. 11) to maximize the strain energy. Again, the optimum design indicates a trend towards multiple microscale actuators. Although these actuators cannot be tested currently, ongoing research in the microscale community will allow, we hope, the development of these actuators in the near future.

\section{Concluding Remarks}

The piezoelectric material PVDF was used to construct a C-block type actuator. This single actuator was used as a building block to form a better actuator by combining multiple units in series and in parallel that gives significantly superior performance. Experimental validation of the mathematical model was provided. Since efficient design of these actuators involves various performance issues, an optimization technique was used to investigate design trends. The associated design variables were both continuous and discrete in nature. Therefore, a hybrid optimization technique was used that is based on a SA algorithm and utilizes gradient information for improved efficiency. Deflection, force, and strain energy were used as objective functions with constraints on geometry and voltage. The following important observations were made from this study.

1) Experimental tests conducted show excellent agreement with the C-block actuator mathematical model

2) The hybrid optimization procedure significantly improves design objectives while satisfying all constraints.

3) The ratio of the piezoelectric layer to the total actuator thickness approaches a value of 0.4 , which was previously determined to be an optimal thickness ratio by other researchers for straight bimorph configurations.

4) Maximization of the strain energy results in an optimum configuration that shows a distinct tradeoff between maximization of deflection and force separately. The optimum configuration comprises multiple thin actuators with many parallel and series units and an intermediate value of radius.

5) The procedure results in a C-block actuator configuration that provides more work than an equivalent straight bimorph actuator, indicating a better design choice.

6) The optimal designs indicate a need for the development of microscale technology.

\section{Acknowledgments}

This research was supported by the U.S. Army Research Office, Grant DAAH04-94-G-0157, technical monitor Gary Anderson.

\section{References}

${ }^{1}$ Crawley, E. F., and de Luis, J., "Use of Piezoelectric Actuators as Elements of Intelligent Structures," AIAA Journal, Vol. 5, No. 10, 1987, pp. 1373-1385.

${ }^{2}$ Heeg, J., "Flutter Suppression via Piezoelectric Actuation," M.S. Thesis, George Washington Univ., Washington, DC, Aug. 1991.

${ }^{3}$ Samak, D. K., and Chopra, I., "Design of High Force, High Displacement Actuators for Helicopter Rotors," Proceedings of the SPIE North American Conference on Smart Structures and Materials (Orlando, FL), 1994 pp. 86-89.

${ }^{4}$ Chen, P. C., and Chopra, I., "Induced Strain Actuation of Composite Beams and Rotor Blades with Embedded Piezoceramic Elements," Proceedings of the SPIE North American Conference on Smart Structures and Materials (Orlando, FL), 1994, pp. 123-140.

${ }^{5}$ Thirupathi, S. R., and Naganathan, G. G., "Use of Piezoceramic Actuator for Automotive Active Suspension Mechanisms: A Feasibility Study," Proceedings of the 22nd Biennial Mechanism Conference (Scottsdale, AZ), 1992,pp. 233-241.

${ }^{6} \mathrm{Baz}, \mathrm{A}$, and Poh, "Performance of an Active Control System with Piezoelectric Actuators," Journal of Sound and Vibration, Vol. 126, No. 2, 1988 , pp. 327-343.

${ }^{7}$ Brei, D., "Design and Development of a New Class of Piezoelectric Actuators for Force Improvement," Proceedings of the Society of Engineering Science 31st Annual Technical Meeting (College Station, TX), 1994.

${ }^{8}$ Brei, D., "Design and Development of a Piezoelectric Microactuator Building Block for Deflection Improvement," Proceedings of the Symposium on Micro-Mechanical Systems, ASME Winter Annual Meeting (Chicago, IL), Vol. 2, 1994, pp. 717-723.

${ }^{9}$ Vanderplaats, G. N., Numerical Optimization Techniques for Engineering Design with Applications, McGraw-Hill, New York, 1984.

${ }^{10}$ Onoda, J., and Hanawa, Y., "Optimal Locations of Actuators for Statistical Static Shape Control of Large Space Structures: A Comparison of Approaches," Proceedings of the 33rd AIAA/ASME/ASCE/AHS/ASC Structures, Structural Dynamics, and Materials Conference (Dallas, TX), AIAA Washington, DC, 1992, pp. 2788-2795.

${ }^{11}$ Goldberg, D. E., Genetic Algorithms in Search, Optimization and Machine Learning, Addison-Wesley, New York, 1989.

${ }^{12}$ Chattopadhyay, A., and Seeley, C. E., "Development of a Hybrid Optimization Technique with Application to Buckling of Cylindrical Shells," Proceedings of WCSMO-1, The First World Congress of Structural and Multidisciplinary Optimization (Goslar, Lower Saxony, Germany), 1995.

${ }^{13}$ Kirkpatrick, S., Gelatt, C. D., Jr., and Vecchi, M. P., "Optimization by Simulated Annealing," Science, Vol. 220, No. 4598, 1983, pp. 671-680.

${ }^{14}$ Lombardi, M., Haftka, R. T., and Cinquini, C., "Optimization of Composite Plates of Buckling by Simulated Annealing," Proceedings of the 31st AIAA/ASME/ASCE/AHS/ASC Structures, Structural Dynamics, and Materials Conference (Dallas, TX), AIAA, Washington, DC, 1992. pp. 2552-2562.

${ }^{15}$ Crawley, E. F., and Anderson, E. H., "Detailed Models of Piezoelectric Actuation of Beams," Proceedings of the 30th AIAA/ASME/ASCE/AHS/ASC Structures, Structural Dynamics, and Materials Conference (Mobile, AL) AIAA, Washington, DC, 1989, pp. 2000-2010.

${ }^{16}$ Tzou, H. S., "Development of a Lightweight Robotic End Effector Using Polymeric Piezoelectric Bimorph," IEEE Conference on Robotics and Automation, 1989, pp. 1704-1709.

${ }^{17}$ Kreisselmeier, G., and Steinhauser, R., "Systematic Control Design by Optimizing a Vector Performance Index," Proceedings of the International Federation of Active Controls Symposium on Computer-Aided Design of Control Systems (Zurich, Switzerland), 1979, pp. 107-113.

${ }^{18}$ Seeley, C. E., and Chattopadhyay, A., "A Simulated Annealing Technique for Multiobjective Optimization of Intelligent Structures," Smart Máterials and Structures, Vol. 3, 1994, pp. 98-106.

${ }^{19}$ Anderson, E. H., and Hagood, N. W., "A Method for Improving Randomized Search Placement Algorithms," Proceedings of the SPIE North American Conference on Smart Materials and Structures, 1994, pp. 308-322. 